\section{Isolation of a Mixed Disulphide of Glutathione and Cysteinylglycine from a Partial Hydrolysate of Glutathione}

GLutathrone, $\gamma$-glutamylcysteinylglycine, can easily be split into glutamic acid and cysteinylglycine by mild acid hydrolysis ${ }^{1}$, and even without acid by heating in a water solution ${ }^{2}$. The tripeptide has therefore been used as material for the preparation of cysteinylglycine. In order to prepare this dipeptide for a microbiological purpose, the latter of the two methods was tried.

Glutathione, dissolved in distilled water, was hydrolysed for five days at $62^{\circ} \mathrm{C}$. The cysteinylglycine and possibly unhydrolysed glutathione were precipitated as their mercaptides with mercuric sulphate. The precipitate was washed with water and decomposed with gaseous hydrogen disulphide. The mercuric sulphide was removed by centrifugation and the supernatant neutralized with barium hydroxide. After evaporation of the solution to a few millilitres, precipitation was made with absolute alcohol. During storage the SH-groups originally present were oxidized (test with sodium nitroprusside). In order to examine whether the hydrolysis was complete, a one-dimensional descending chromatogram was developed in the usual manner with acetic acid : $n$-butanol : water $(1: 4: 5)$ and Whatman No. 1 paper. More than one component was detected, but the resolution of the mixture was unsatisfactory, and therefore electrophoresis on paper was tried according to the method of Kunkel and Tiselius ${ }^{3}$. The run was made in acetate buffer $(p H 4 \cdot 5, \mu 0 \cdot 05)$ with about $0.2 \mathrm{mgm}$. of the substance on Munktell 20 paper and using a potential gradient of $5 \mathrm{~V} . / \mathrm{cm}$. for six hours. The position of the material was determined by treating the paper with ninhydrin solution. As seen from the illustration (row No. 2), three spots could be detected on heating the paper, two of which ( $B$ and $C$ ) travelled towards the anode, while the third (A) was immobile. Spot $C$ was found to be oxidized glutathione, since this substance gave an identical spot (row No. 1 in the illustration). Judging from its yellowish colour and position, spot $A$ was cystinylbisglycine, and this was confirmed by paper chromatography.

Spot $B$ was eluted from a paper run in the same way as above, its position being determined with ninhydrin on a narrow strip of the paper. After hydrolysis of the component in $6 \mathrm{~N}$ hydrochloric acid at $110^{\circ} \mathrm{C}$. overnight, a paper chromatogram revealed the presence of the same amino-acids as in glutathione. Since no SH-groups were present, the component must be a disulphide. It was therefore oxidized with performic acid and chromatographed on Whatman No. I paper with acetic acid: $n$-butanol : water $(1: 4: 5)$. Two spots were detected, the faster one being $\left(\mathrm{CySO}_{3} \mathrm{H}\right)$. Gly and the slower one, $\gamma$-Glu. $\left(\mathrm{CySO}_{3} \mathrm{H}\right)$. Gly, the sulphonic acid derived from glutathione. Synthetic samples of these peptides were used as references. 'The unknown compound

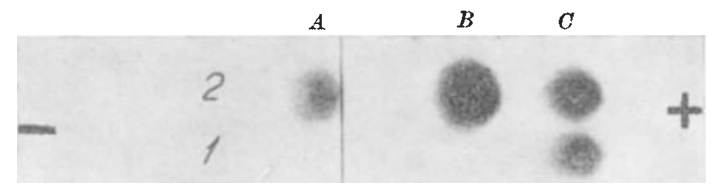

Electrophoresis on paper of a partial hydrolysate of glutathione : (1) oxidized glutathione; (2) partial hydrolysate of glutathione was thus a mixed disulphide of glutathione and cysteinylgly cine,

$$
\begin{gathered}
\gamma \text {-Glu.(CyS).Gly } \\
\text { । } \\
\text { (CyS).Gly. }
\end{gathered}
$$

Since both the glutathione and the cysteinylglycine had been isolated in their reduced forms from the hydrolysis mixture, this mixed disulphide must in this case have been formed secondarily by oxidative coupling of the peptides during storage. It might also be formed as an intermediate product in the hydrolysis of oxidized glutathione. A similar mixed disulphide has also been prepared from glutathione and cystein by oxidation with perhydrol at $p \mathbf{H} 7 \cdot 5$. It could be separated from the reaction mixture in the same way as above.

\section{Biokemiska Institutionen, Uppsala. \\ June 8.}

${ }^{2}$ Olson, C. K., and Binkley, F., J. Biol. Chem., 186, 731 (1950). ' Kendall, E. C., Mason, H. L., and McKenzie, B. F., J. Biol. Chem.,
88, $402(1930)$.

${ }^{3}$ Kunkel, H. G., and Tiselius, A., J. Gen. Physiol., 35, 89 (1951).

- Sanger, F., Biochem. J., 44, 126 (1949).

\section{Enzymatic Hydrolysis of Urinary Neutral 17-Ketosteroid Conjugates}

Ir is generally accepted that urinary steroids are excreted almost entirely in the form of watersoluble conjugates with glucuronic and sulphuric acids. Acid hydrolysis has been extensively used to liberate free steroids from these conjugates; but the conditions necessary for complete hydrolysis are such that considerable changes occur within the steroid molecules themselves, particularly the substitution of the hydroxyl group of androst-5-en-3- $\beta$-ol-17-one (dehydroisoandrosterone) by chlorine.

$\beta$-Glucuronidase preparations have been shown to split steroid glucuronides and have been applied to the hydrolysis of corticosteroid, œstrogen and ketosteroid conjugates. The application of sulphatase concentrates has been mainly confined to the liberation of the phenolic œstrogens from their sulphates; the sulphatases of microbiological origin have been shown to be ineffective for hydrolysing the neutral 17-ketosteroid conjugates'. Henry and 'Thevenet ${ }^{2}$ and Henry, Thevenet and Jarrige ${ }^{3}$ have, however, recently described the total hydrolysis of urinary steroid conjugates and the hydrolysis of the potassium salt of androst-5-en-3- $\beta$-ol-17-one sulphate with sulphatase and $\beta$-glucuronidase preparations from the edible snail (Helix pomatia).

The recently published work of Dodgson and Spencer ${ }^{4}$ has shown the high $\beta$-gluaturonidase and phenol sulphatase activity of extracts of the viscera.] hump of the common limpet (Patella vulgata). We have found that an extract of the visceral hump of this species of mollusc hydrolysed a sufficiently large amount of the neutral 17-ketosteroid conjugates present in urine to indicate that a steroid alcohol sulphatase was active. The extract was prepared from acetone-dried visceral hump by extraction with $0 \cdot 1 \mathrm{~N}$ potassium chloride at $p \mathrm{H} 8.0$, precipitation by half saturation with ammonium sulphate, followed by dialysis and freeze-drying of the precipitate.

This extract, when incubated with normal male urine at $p \mathrm{H} 5.0$ for periods up to $96 \mathrm{hr}$., released amounts of 17-ketosteroids exceeding 90 per cent of 